A N N A L E S Annales de Bretagne et des Pays de l'Ouest

\title{
Les pupilles d'Ille-et-Vilaine morts pour la France
}

\section{Martine Fauconnier Chabalier}

\section{OpenEdition}

\section{Journals}

Édition électronique

URL : https://journals.openedition.org/abpo/6618

DOI : $10.4000 / a b p o .6618$

ISSN : 2108-6443

\section{Éditeur}

Presses universitaires de Rennes

\section{Édition imprimée}

Date de publication : 10 décembre 2020

Pagination : 229-231

ISBN : 978-2-7535-8225-5

ISSN : 0399-0826

\section{Référence électronique}

Martine Fauconnier Chabalier, "Les pupilles d'Ille-et-Vilaine morts pour la France », Annales de Bretagne et des Pays de l'Ouest [En ligne], 127-4 | 2020, mis en ligne le 10 décembre 2020, consulté le 06 janvier 2023. URL : http://journals.openedition.org/abpo/6618; DOI : https://doi.org/10.4000/abpo 6618 
et du district, sur les territoires des communes, enfin sur la mise en place de la Constitution civile du clergé. Lorient ne tarde pas à s'affirmer comme " le leader de la Révolution et son gendarme dans le Morbihan " (p. 102), dans un département qui embrasse largement le camp de la contre-Révolution.

Dans le quatrième et dernier chapitre - « La pérennité du ressentiment entre le pays de Lorient (Lorient-Port-Louis-Pleomeur-Hennebont) et Vannes : une fracture politique durable ravivée dans la seconde moitié du XIX siècle" (p. 105-123) - l'auteur montre que si les ressentiments des communes proches de Lorient tendent à s'atténuer, grâce à la dynamique économique engendrée par la révolution sardinière, la croissance de l'industrie métallurgique et l'essor du balnéaire, c'est loin d'être le cas à Vannes qui certes conserve sa prééminence administrative, mais reste largement figée dans ses structures héritées de l'Ancien Régime. Les tentatives d'implantation de grands ports militaires et de commerce dans le golfe du Morbihan datant de la fin du XIX ${ }^{\mathrm{e}}$ siècle et du début du Xxe siècle, comme celle d'obtenir la création d'une chambre de commerce à Vannes, témoignent d'une volonté de changer profondément les choses, mais ces projets sont jugés " chimériques " par Gérard Le Bouëdec (p. 113).

En dernière analyse, comprendre la construction de ces ressentiments implique, selon l'auteur, de "se pencher sur les rapports de forces" (p. 130). Pour lui, Lorient incarne l'offensive d'un " nouveau monde mondialisé puis inséré dans la révolution industrielle ", tandis que les autres sites sont systématiquement sur la défensive face à cette ville qui peut compter sur le soutien de l'État et sur une élite entrepreneuriale très active.

La lecture de ce stimulant ouvrage appellerait des prolongements, d'abord chronologiques : pourquoi arrêter l'étude en 1914, alors que la nouvelle histoire portuaire de Lorient s'achève en 2016 et que l'exemple de la création de l'université de Bretagne Sud, cité par Gérard Le Bouëdec dans son introduction, illustre un rapprochement entre Lorient et Vannes? Même si chaque cas est par définition unique, cette étude doit ensuite susciter des approches comparatives, en premier lieu avec les autres ports-arsenaux français, à l'image de Brest dans son rapport avec Quimper et le Finistère ou de Cherbourg dans ses relations avec Saint-Lô et la Manche. N'est-ce pas là un beau sujet pour un futur volume?

Bernard MichON

1914-1918. Les pupilles d'Ille-et-Vilaine morts pour la France, Rennes, Archives départementales d'Ille-et-Vilaine, coll. " Patrimoine écrit d'llle-et-Vilaine ", novembre 2019, 165 p.

De nombreuses cérémonies ont été organisées à l'occasion du centenaire de la Première Guerre mondiale. L'une d'entre elles, particulièrement émouvante, a rendu hommage aux pupilles et anciens pupilles de l'Assistance Publique d'Illeet-Vilaine, " morts au champ d'honneur et disparus ". En souvenir de ces soldats, deux plaques ont été dévoilées au Conseil départemental le 9 novembre 2018. La plus ancienne, qui date de 1921, portait quatre-vingts noms. La seconde en a mis en lumière quatre-vingt-cinq autres. 1914-1918, les pupilles d'Ille-et-Vilaine morts pour la France, paru en novembre 2019, fait suite à cette manifestation. Ce troisième volume de la collection Patrimoine écrit d'Ille-et-Vilaine est un travail collectif des Archives départementales et du service Adoption et accès aux données personnelles de la direction Enfance Famille. 
Dans cet ouvrage, un livre d'or présente chacun des 165 pupilles avec leur nom, prénom, date et lieu de naissance ainsi que la date d'admission à l'Assistance Publique et la catégorie juridique. Il précise aussi leur grade et leur régiment au moment de leur décès. Sont également mentionnés le lieu d'inhumation lorsqu'il est connu, les monuments aux morts où figure leur nom, les citations et décorations éventuelles. Ces jeunes ont été élevés à l'école de la République où on leur a inculqué le sentiment de l'honneur. Il n'est donc pas étonnant que certains soient des engagés volontaires. La première plaque commémorative avait d'ailleurs été posée "pour l'édification des pupilles".

Une recherche approfondie a permis de brosser vingt-trois portraits individuels, détaillés et pleins d'empathie. Chaque auteur, avec sa sensibilité propre et selon la teneur des documents recueillis, retrace l'enfance et la vie de jeune adulte et de soldat du pupille disparu. Certains ont une histoire particulière comme Alphonse Descours, dont on découvre qu'il a été placé chez une nourrice sous un faux nom. À ses vingt ans, devant l'impossibilité de retrouver sa véritable identité, il est déclaré " né à Rennes de père et mère inconnus ". La plupart des autres reflètent la vie de nombre de pupilles. Louis Dévédec se retrouve abandonné suite au décès de sa mère et à la disparition de son père. Il est placé chez une nourrice à Corps-Nuds où il reste jusqu'à l'âge de douze ans. Sa santé est bonne, son caractère et sa moralité ne posent pas de problème. Bon élève, il obtient son certificat d'études élémentaires. Il est alors placé comme domestique de " maison bourgeoise ", ce qui est un privilège, chez un médecin à Paris début décembre 1905. Son emploi du temps nous est relaté : lever à six heures (à 6 h 30 l'hiver), brossage des vêtements et des chaussures du maître de maison, petit-déjeuner (café au lait), puis ménage de l'antichambre du cabinet et du salon jusqu'à $10 \mathrm{~h} 45$. Il met ensuite le couvert pour le déjeuner et, de $13 \mathrm{~h} 30$ à 17 heures, se tient à disposition dans l'antichambre pour accueillir les clients. À 18 h 45 il met le couvert et sert le dîner. Il se couche à 21 h 30 sauf lorsque Monsieur et Madame sont au théâtre. Il est libre un dimanche sur deux, de $13 \mathrm{~h}$ à $18 \mathrm{~h} 30$. Mais estimé trop jeune pour répondre aux besoins des patrons, il est finalement renvoyé en Bretagne, un mois plus tard. Victor Geslin, "gentil garçon ", est plus classiquement domestique agricole après son certificat d'études. "C'est un bon travailleur ", dont tout le monde est content. À dix-sept ans, il est placé chez un agriculteur où il se trouve " comme un fils chez son père ". Il reviendra chez lui en permission, même après sa majorité. "Soldat très courageux faisant partie d'un groupe d'éclaireurs ", il est muté début 1918 chez les tirailleurs indigènes comme caporal. Il est tué le 12 août. On découvre aussi que les jugements portés sur certains jeunes pupilles ne sont guère amènes, surtout pour les moralement abandonnés qu'un agent de l'AP décrit sans discernement comme des "éléments difficiles, vicieux ou délinquants ", " qui ont vécu jusqu'à un âge avancé dans un milieu contaminé à l'école du vice et de la prostitution ". Ainsi Émile Lafosse est dit : "Sournois ", " entêté ", " arriéré, grossier, insolent " et jugé " pauvre garçon d'intelligence très bornée ". Dans le jugement de déchéance paternelle, sa mère avait été dépeinte comme une " mauvaise mère " qui ne peut que donner " de mauvais conseils et de déplorables exemples ". Émile part au front dès le 5 août 1914. Blessé à deux reprises, il meurt en 1915 dans la bataille de Champagne. Le monument aux morts de Vieuvy-sur-Couesnon porte son nom.

Au-delà de ces biographies particulièrement intéressantes, des données d'ensemble sont apportées sur ces hommes et aussi sur l' Assistance Publique d'Ille-etVilaine. Il est notamment relaté les fondements des lois Strauss des 27 et 28 juin de 1904, lois qui rénovent l'Assistance Publique et retirent les prérogatives des Commissions administratives des hospices au bénéfice des préfets. Il est également brossé un portrait très vivant de l'inspecteur Émiland Cannet qui dirigea les services de l' Assistance Publique d'Ille-et-Vilaine de juillet 1904 à fin décembre 1923 et 
fut un correspondant assidu des pupilles pendant la Guerre.

On apprend aussi que parmi les 165 pupilles morts pour la France, 84 soit la moitié ont été des enfants abandonnés, 51 des moralement abandonnés (30,1\%) et 26 des orphelins (15,8\%), alors même que les nouveaux admis en 1900 sont pour les trois quarts des enfants abandonnés et que les moralement abandonnés ne représentent que 13,5\% des pupilles et les orphelins 10,5\%. L'âge d'entrée à l'Assistance Publique apporte aussi de précieux renseignements. Seuls $4 \%$ des soldats ont été accueillis avant un mois et $12 \%$ entre un mois et un an. On sait pourtant par ailleurs que parmi les enfants abandonnés, de loin les plus nombreux des pupilles, huit sur dix arrivent avant un mois. Ce décalage avec les données relatives aux soldats décédés soulignent combien était importante la mortalité infantile des enfants trouvés et abandonnés, pupilles arrivés très tôt à l'Assistance Publique. Il est rappelé qu'après leurs premières années en nourrice, les pupilles de l'époque sont gagés dès treize ans, généralement comme pâtres puis domestiques agricoles. 134 des 165 pupilles exercent d'ailleurs un métier agricole. Il est aussi souligné combien l'Assistance Publique se veut gardienne du secret : les origines ne sont pas révélées et il n'est pas permis de correspondance avec la famille, même pendant la guerre.

Les pupilles et anciens pupilles ont payé un lourd tribut à la Nation. Le plus jeune est mort à dix-huit ans, le plus âgé à quarante-huit. La très grande majorité des soldats honorés ont fait la guerre comme hommes de troupe. Presque tous appartiennent à l'infanterie, arme la plus décimée dans le conflit. En 1919, l'inspecteur recensait sur 380 pupilles et anciens pupilles mobilisés, 65 morts au champ d'honneur, soit $21 \%$ de tués, 110 blessés, 15 disparus et 22 faits prisonniers.

La reproduction de documents enrichit agréablement les textes, tout au long de l'ouvrage et dans l'annexe "illustration ». Il s'agit en particulier de lettres et de cartes postales adressées à l'inspecteur. L'Assistance avait organisé un service spécial pour la correspondance avec les pupilles militaires. Certains courriers sont assez convenus et portent sur la demande d'un pécule ou le remerciement de son octroi. D'autres se rapprochent davantage d'une lettre personnelle adressée à un proche. Certains adoptent un ton très guerrier : " Je me suis engagé pour tuer et éventrer la chair non civilisée ", écrit l'un; "Je vous assure que les boches reçoivent quelque chose comme pruneau ", témoigne un autre. D'autres courriers évoquent davantage les sentiments éprouvés : "La guerre est longue surtout pour nous qui n'avons ni parents ni aucune ressource ". D'autres encore, ou les mêmes, font part des conditions matérielles, comme par exemple le manque de cidre. Des photos nous rendent quelques-uns encore plus présents. Certaines comme celle d'Édouard Bandily ont été prises alors qu'ils étaient enfants. Mais les autres nous les montrent en soldats, à l'exemple de celle de Louis Dévédec en uniforme de zouave ou d'Eugène Bunel qui pose en août 1917. Yves Raimbault, quant à lui, arbore sa croix de guerre.

Cet ouvrage plein d'enseignements et très vivant est un très bel hommage à ces jeunes hommes et à leur courage.

Martine FAUCONNIER CHABALIER

Catala, Michel (dir.), Les poches de l'Atlantique 1944-1945. Le dernier acte de la Seconde Guerre mondiale en France, Rennes, Presses universitaires de Rennes, coll. « Histoire », 2019, 324 p.

Le " front des oubliés ", c'est ainsi que dans le sud de la Bretagne l'on nomme les combats ayant eu lieu pour libérer les poches de l'Atlantique. L'ouvrage qui lui 